\title{
Peak nasal inspiratory flow: uma possível ferramenta para a motricidade orofacial?
}

\author{
Peak nasal inspiratory flow: a possible instrument in orofacial \\ myology?
}

\author{
Andréa Rodrigues Motta ${ }^{1}$, Silvana Bommarito ${ }^{2}$, Brasília Maria Chiari ${ }^{3}$
}

\begin{abstract}
RESUMO
Os fonoaudiólogos que atuam no campo da Motricidade Orofacial atendem com frequência, pacientes com obstrução nasal; entretanto, nem sempre o acesso ao profissional responsável pelo diagnóstico otorrinolaringológico é fácil. O Peak Nasal Inspiratory Flow é um instrumento barato, de fácil manuseio, amplamente citado na literatura internacional, que tem por objetivo avaliar a patência nasal. $\mathrm{O}$ objetivo do presente estudo foi realizar uma revisão da literatura acerca do Peak Nasal Inspiratory Flow, fornecendo subsídios para reflexões acerca de seu emprego nos distúrbios miofuncionais orofaciais e cervicais. Para tanto, foi realizada uma revisão da literatura nas bases de dados Medline, Cochrane Library, LILACS e SciELO empregando-se os descritores peak, inspiratory, nasal e flow. O Peak Nasal Inspiratory Flow mostrou-se uma técnica simples, barata, validada e com boa reprodutibilidade. Parece, portanto, tratar-se de um instrumento útil para avaliação da patência nasal, apesar de algumas limitações, fornecendo dados complementares ao diagnóstico miofuncional orofacial e cervical. Entretanto, estudos clínicos precisam ser conduzidos para que se comprove ou refute a hipótese.
\end{abstract}

Descritores: Técnicas de diagnóstico do sistema respiratório; Permeabilidade; Obstrução nasal/fisiopatologia; Respiração bucal; Cavidade nasal/fisiologia

\section{INTRODUÇÃO}

A Motricidade Orofacial cada vez mais tem buscado métodos de avaliação instrumental, especialmente com o objetivo de obter dados complementares às avaliações clínicas.

Existem vários métodos de investigação das vias áreas, não havendo, entretanto, um padrão que consiga abranger todas as dimensões. Alguns métodos podem ser classificados em anatômicos, já que avaliam a dimensão estrutural; em fisiológicos, mensurando um parâmetro funcional (ou biológico) e em subjetivos, que levam em consideração o relato ou experiência do indivíduo ${ }^{(1)}$.

A avaliação subjetiva dos sintomas, por meio de ques-

Trabalho realizado no Departamento de Fonoaudiologia da Universidade Federal de São Paulo - UNIFESP - São Paulo (SP), Brasil.

(1) Pós-graduanda (Doutorado) em Distúrbios da Comunicação Humana pelo Departamento de Fonoaudiologia da Universidade Federal de São Paulo UNIFESP - São Paulo (SP), Brasil; Professora Assistente do Departamento de Fonoaudiologia da Universidade Federal de Minas Gerais - UFMG - Belo Horizonte (MG), Brasil.

(2) Doutora, Professora Adjunto do Departamento de Fonoaudiologia da Universidade Federal de São Paulo - UNIFESP - São Paulo (SP), Brasil.

(3) Doutora, Professora Titular do Departamento de Fonoaudiologia da Universidade Federal de São Paulo - UNIFESP - São Paulo (SP), Brasil.

Endereço para correspondência: Andréa Rodrigues Motta. R. João Chagas, 333/1002, União, Belo Horizonte (MG), Brasil, CEP: 31170-370. E-mail: andreamotta@ufmg.br

Recebido em: 10/6/2009; Aceito em: 21/12/2009 tionários, é importante tanto para o diagnóstico quanto para pesquisas, entretanto, nem sempre métodos objetivos e subjetivos de avaliação da obstrução nasal se correlacionam ${ }^{(2)}$. Os métodos objetivos mais comumente empregados são: os medidores de fluxo inspiratório nasal, a rinometria acústica e a rinomanometria anterior ${ }^{(3)}$. Entretanto, esses dois últimos exames requerem equipamentos caros e complexos limitando seu uso na clínica ${ }^{(2)}$.

A rinometria acústica emprega a verificação da reflexão da onda sonora para medir a relação entre a distância dentro da cavidade nasal e a área de secção transversal. A onda sonora refletida é convertida em um gráfico, sendo útil também para a localização de obstruções na cavidade nasal ${ }^{(4)}$. Seu emprego é limitado por depender de um técnico treinado, apresentar custo elevado e demandar tempo para realização $0^{(5)}$.

Já a rinomanometria avalia a resistência nasal por meio da relação entre o fluxo aéreo transnasal e a pressão necessária para alcançar esse fluxo ${ }^{(4,6)}$. As medidas são realizadas durante as duas fases do ciclo respiratório e em ambas as fossas nasais ${ }^{(3)}$. Dependendo da técnica empregada, pode fornecer a informação de cada narina separadamente ou uma medida integrada das duas narinas ${ }^{(6)}$. Trata-se de um equipamento relativamente caro, complexo ${ }^{(6,7)}$ e que demanda certo tempo na execução do exame, especialmente em crianças $^{(7)}$. Sua utilização requer cooperação e coordenação do paciente, sendo indicado para estudos que não envolvam grandes amostras ${ }^{(6)}$. 
Uma possibilidade a ser investigada nessa vertente é o Peak Nasal Inspiratory Flow (PNIF): um método não invasivo, barato, de fácil manuseio e obtenção no mercado e comumente utilizado para avaliar a patência nasal, ou seja, a permeabilidade da via aérea superior.

Trata-se de uma medida fisiológica que indica o pico de fluxo nasal, em litros por minuto, atingido durante uma inspiração forçada, exclusivamente nasal ${ }^{(1,2,6-9)}$. A avaliação é realizada com uma máscara de silicone acolchoada que abrange as regiões oral e nasal acoplada a um tubo de acrílico ${ }^{(6)}$, como pode ser observado nas Figuras 1 e 2.

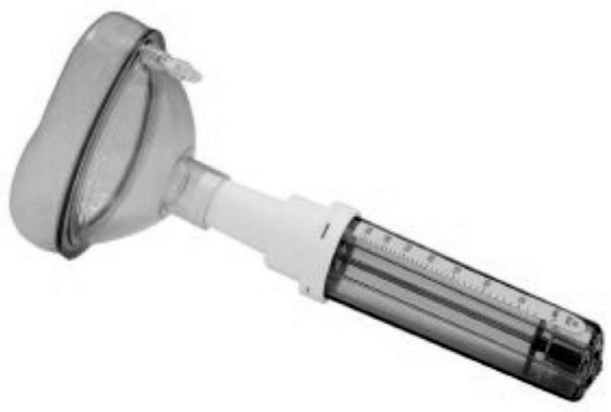

Figura 1. In-Check Nasal®, modelo de PNIF da Clement Clarke International

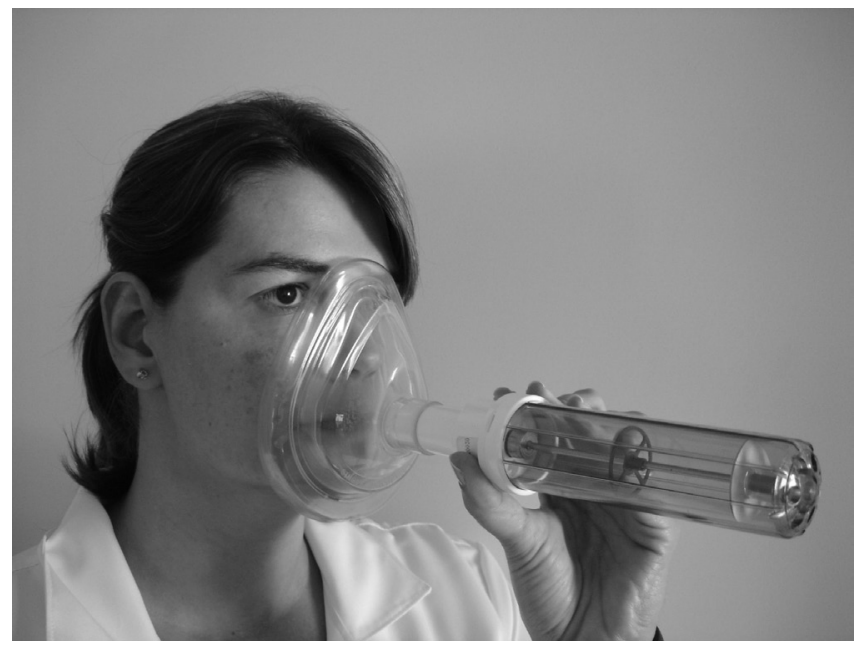

Figura 2. Demonstração do uso do equipamento

A sensação de obstrução apresenta diversas etiologias e depende não só da dimensão da cavidade nasal e do fluxo nasal, bem como da presença de secreção e dos receptores de pressão, calor, dor, dentre outros. Assim, não se justifica descrever a obstrução apenas pelo espaço e fluxo nasais ${ }^{(8)}$. Entretanto, buscar de alguma maneira quantificar a obstrução é um fator relevante, uma vez que esta mensuração auxilia no diagnóstico acurado, na definição do estágio de uma doença e na avaliação dos resultados terapêuticos ${ }^{(8)}$.

Com base em uma revisão da literatura, este estudo teve como objetivo apresentar algumas considerações acerca do Peak Nasal Inspiratory Flow oferecendo subsídios para que os fonoaudiólogos possam refletir sobre seu emprego.

Para tanto, foi realizada entre julho e dezembro de 2008 uma pesquisa bibliográfica abrangendo publicações dos últimos cinco anos, empregando-se os descritores peak, inspiratory, nasal e flow. A associação desses no Medline (via PubMed) indicou a existência de 97 artigos. Para busca no LILACS (via Bireme) e SciELO, em decorrência dos poucos estudos encontrados, empregou-se apenas os unitermos peak e flow, tendo-se localizado em cada uma das bases de dados 49 e 81 artigos, respectivamente.

A partir da análise dos títulos e leitura dos resumos foram selecionados os artigos que se adequavam ao objetivo de apresentar a aplicabilidade, funcionamento e limitações do Peak Nasal Inspiratory Flow.

\section{REVISÃO DA LITERATURA}

O Peak Nasal Inspiratory Flow é considerado um índice indireto da obstrução nasal, uma vez que aumentos na resistência nasal modificam o fluxo aéreo nasal e consequentemente o pico de fluxo inspiratório ${ }^{(6)}$. Pode ser utilizado por médicos no diagnóstico de alterações nasais e na verificação da eficácia de um tratamento, bem como na atenção primária, por outros profissionais, como método diagnóstico e de follow-up nos casos de alterações nasais ${ }^{(7)}$.

Seu uso é amplamente relatado na literatura internacional, sendo muito utilizado em pesquisas médicas para a avaliação da eficácia de medicações/tratamentos ${ }^{(10-13)}$, bem como da influência hormonal sobre a patência nasal ${ }^{(14-16)}$. Trata-se do único método conveniente para o monitoramento doméstico, avaliando a variação circadiana ou os efeitos do ambiente na patência nasal ${ }^{(6)}$.

$\mathrm{Na}$ área da Fisioterapia, O PNIF foi recentemente empregado com o objetivo de avaliar o impacto de um programa de treinamento muscular ventilatório (TMV) e da respiração nasal (TRN) na força muscular ventilatória, bem como no fluxo inspiratório nasal de crianças respiradoras orais. Para tanto, foram acompanhados oito participantes com idade entre cinco e 12 anos, tendo-se empregado para obtenção do pico de fluxo inspiratório nasal uma adaptação do instrumento In-Check Inspiratory Flow Meter (Clement Clarke), a saber, dispositivos nasais bilaterais. O programa de treinamento foi constituído por 15 sessões de 60 minutos com frequência de três vezes por semana, tendo sido o TMV realizado no nasomanômetro e o TRN conduzido concomitantemente, assim como atividades que exigissem o modo respiratório nasal. De acordo com a análise dos dados o programa de treinamento muscular ventilatório concomitante a um treinamento da respiração nasal proporciona melhora na força muscular ventilatória bem como no fluxo respiratório nasal de crianças respiradoras orais ${ }^{(17)}$.

Dentre 228 otorrinolaringologistas franceses que aceitaram testar o PNIF em pacientes alérgicos, $65 \%$ o utilizaram de forma sistemática e consideraram como principais vantagens do método a objetivação do sintoma de obstrução e a possibilidade de uma medida quantitativa desta obstrução ${ }^{(18)}$. Estudos que avaliaram a aprendizagem na utilização do método por parte de estudantes de Medicina ${ }^{(19)}$ e pacientes ${ }^{(18)}$ identificaram facilidade no manuseio do equipamento.

Assim, pode-se observar que o PNIF tem sido descrito como uma técnica fácil e útil para se verificar a extensão da 
obstrução nasal ${ }^{(2,5)}$, sendo seu uso indicado na atenção primária e secundária ${ }^{(7)}$.

O pico de fluxo respiratório pode ser medido tanto na inspiração quanto na expiração e estes dois dados se correlacionam em indivíduos sem problemas respiratórios ${ }^{(20)}$. Em casos de pacientes asmáticos, o Peak Expiratory Flow (PEF) apresenta melhor aplicabilidade ${ }^{(21)}$. Entretanto, o PNIF é a técnica melhor validada para monitoramento em casa de ensaios $\operatorname{clínicos}^{(6)}$, além do ato inspiratório ser considerado mais reprodutível do que o expiratório ${ }^{(4)}$. O PNIF também apresenta vantagens em relação ao PEF acerca da higienização, uma vez que os instrumentos são limpos apenas com álcool ${ }^{(7)}$.

$\mathrm{Na}$ literatura é possível observar o interesse por parte dos fonoaudiólogos da área de voz no PEF. No Brasil, uma pesquisa investigou a correlação entre o PEF e o tempo máximo de fonação em pacientes asmáticos, tendo constatado uma proporcionalidade entre as variáveis ${ }^{(22)}$.

Diferentes modelos de PNIF são citados em diversos estudos, tais como In-check nasal ${ }^{(2,5)}$, In-check dial ${ }^{(8)}$,Youlten peak flow meter ${ }^{(7,20,23)}$ (Clement Clarke International); In-check nasal flow meter ${ }^{(1)}$ (Alliance Tech Medical Inc); In-check inspiratory flow-meter ${ }^{(9)}$ (Ferdiand Menzel Medizintechnik GmbH). Deve-se ter especial atenção ao tamanho da máscara a ser empregada, uma vez que a mesma precisa ser firmemente ajustada na face do paciente, entretanto, sem pressionar o nariz ${ }^{(6,9,20)}$.

Algumas pequenas variações nos procedimentos empregados para avaliação com o PNIF podem ser observadas na literatura. Instruções claras são fundamentais para que a realização da avaliação seja conduzida de forma correta ${ }^{(1,2,8,9)}$, sendo a supervisão do teste por parte do profissional indispensável ${ }^{(2,9)}$. Alguns autores optam por demonstrar ao paciente o procedimento $^{(1,23)}$ enquanto outros realizam sessões de treinamento ${ }^{(5,9)}$.

O método do volume residual, que consiste em realizar uma inspiração rápida e forçada logo após uma expiração total é o mais empregado ${ }^{(2,7,9,20)}$. Apenas um trabalho ${ }^{(9)}$ especificou o tempo de intervalo entre cada mensuração: 30 segundos, no mínimo.

De uma forma geral três medidas satisfatórias são realizadas, sendo que a maioria dos autores prefere empregar o valor máximo alcançado ${ }^{(2,79,20,23)}$, no lugar da média ${ }^{(8)}$. Apenas um estudo localizado citou empregar as duas possibilidades ${ }^{(1)}$. Em crianças, costumam ser suficientes quatro a cinco medidas para que se alcance o melhor resultado ${ }^{(23)}$.

Alguns autores preferem realizar as medições com o participante posicionado de pé ${ }^{(8,23)}$, sendo que outros optam pela posição sentada ${ }^{(7,19)}$. Cabe ressaltar que a posição corporal pode interferir nos resultados, devendo ser controlada. Dados produzidos de pé tendem a ser maiores do que aqueles obtidos com o participante deitado ${ }^{(1)}$.

Dados de normalidade do PNIF para adultos sem alterações respiratórias foram descritos em um estudo baseado na avaliação de 137 indivíduos entre 16 e 84 anos. Os valores foram apresentados em tabelas levando-se em consideração as variáveis: gênero, idade e altura, fornecendo assim dados auxiliares para a clínica. Cabe ressaltar que a raiz quadrada dos valores mostrou-se um dado mais homogêneo, entretanto, pelo fato do cálculo não fazer parte da rotina ambulatorial, os autores optaram pela discussão dos achados em litros por minuto. De acordo com o estudo, o PNIF tende a diminuir com a idade, tendo-se verificado também uma pequena diferença entre os gêneros. Não foi observada correlação com a altura, apesar de ter apresentado um valor muito próximo ao ponto de corte ${ }^{(7)}$.

Para propor dados de referência para crianças, um estudo avaliou 3170 participantes entre cinco e 18 anos. Meninos apresentaram valores mais altos que meninas, entretanto a diferença só se mostrou significativa após os 12 anos de idade. Os valores do PNIF tenderam a aumentar com a idade, sem que fosse encontrada diferença comparando-se duas idades subsequentes, nos dois gêneros ${ }^{(23)}$.

Considerando-se como padrão principal a avaliação clínica de dois experientes otorrinolaringologistas, outro estudo propôs como valor de referência um ponto de corte para o PNIF no valor de 120 1/min. Para tanto, foram avaliados 53 pacientes com estenose nasal de grau moderado a grave e 40 participantes saudáveis, entre 18 e 60 anos. Um incremento de aproximadamente $35 \%$ do valor do PNIF pôde ser observado como o uso de descongestionantes ${ }^{(9)}$.

Em estudo conduzido com 283 adultos com e sem rinite alérgica, entre 28 e 30 anos, um ponto de corte de $115 \mathrm{l} / \mathrm{min}$, próximo ao acima citado, foi definido para casos de rinite com classificação moderada/grave. A especificidade verificada foi de $72 \%$, a sensibilidade de $65 \%$ e o valor preditivo negativo de $90 \%$, indicando que o PNIF pode ser útil nas situações de screening populacional. Entretanto, em situações ambulatoriais, nas quais a prevalência da rinite tende a aumentar, o valor preditivo negativo tende a ser menor. $\mathrm{O}$ baixo valor preditivo positivo encontrado (34\%) indica que em muitos casos, apesar do valor do PNIF ser reduzido, o indivíduo pode não apresentar rinite moderada/ grave. No estudo citado, o PNIF correlacionou-se com o volume pulmonar, entretanto, não foram observadas associações com altura, peso ou gênero dos indivíduos ${ }^{(2)}$.

A reprodutibilidade do PNIF foi investigada em 283 adultos entre 28 e 30 anos. Três medidas foram obtidas, sendo a primeira menor, estatisticamente, que as demais, sugerindo a ocorrência de um treinamento. Como não se observou diferenças entre a segunda e a terceira mensurações, estas foram consideradas apenas na análise da reprodutibilidade, que se mostrou alta $(0,92)^{(2)}$.

Em outro trabalho de avaliação de reprodutibilidade comparou-se duas medidas realizadas em 528 participantes, 260 homens com idade média de 47 anos e 268 mulheres com média de 44 anos, tendo-se verificado bons resultados. Cabe relatar que a avaliação foi realizada por diferença de médias: PNIF2-PNIF1= 5,51 $\pm 24,931 / \mathrm{min}^{(19)}$.

Valores intersujeitos produzidos pelo PNIF variam consideravelmente ${ }^{(4)}$, sendo que este fato não é explicado pelas variáveis: gênero, idade, altura ${ }^{(7)}$. Entretanto, considerando-se sujeitos antes e após alguma intervenção os dados são altamente confiáveis ${ }^{(4)}$. Cabe ressaltar que diversos estímulos podem influenciar a patência nasal, seja aumentando-a (exercícios físicos e tempo quente) ou diminuindo-a (posição reclinada, dor, ar frio, fumo e hipoventilação). Há relato também de variação diurna nas medidas do PNIF, sendo que pela manhã os valores tendem a ser menores ${ }^{(6)}$.

Diversas publicações comparam o PNIF com outros métodos de avaliação da obstrução nasal ${ }^{(1,2,5,8,9)}$. 
Com o objetivo de investigar a sensibilidade, a especificidade e a acurácia do diagnóstico tanto da rinomanometria anterior quanto do PNIF, 53 pacientes com estenose nasal e indicação cirúrgica e 40 participantes saudáveis foram avaliados, tendo-se como referência a avaliação clínica associada aos exames clínicos. Não foram verificadas diferenças entre a rinomanometria e o PNIF no que diz respeito à sensibilidade $(0,77$ e 0,66 , respectivamente), à especificidade $(0,8$ em ambos os exames) ou a acurácia ( 0,79 e 0,72 , respectivamente). Assim, os dois exames podem ser considerados válidos para uso clínico, ressaltando-se, entretanto, que com os dois métodos aproximadamente $25 \%$ dos pacientes que apresentavam importante quadro obstrutivo, não foram detectados ${ }^{(9)}$.

No mesmo estudo verificou-se que os resultados da rinomanometria e do PNIF não se correlacionaram $(r=0,09)$. Aparentemente os métodos acessam diferentes aspectos do fluxo aéreo nasal. Para os autores as vantagens da rinomanometria incluem a possibilidade de se avaliar as narinas separadamente e a condição mais fisiológica do fluxo aéreo nasal ${ }^{(9)}$.

Um estudo foi conduzido com o objetivo de avaliar a reprodutibilidade do PNIF e da rinometria acústica após teste de estimulação nasal em 14 indivíduos com asma induzida por aspirina. Os exames foram realizados antes e após estimulação em dois momentos distintos, com intervalo de uma semana. As avaliações pré-estimulação não demonstraram diferenças dos métodos entre os diferentes dias. Entretanto, comparando-se os dados antes e após estimulação observou-se que o PNIF demonstrou menor variabilidade ${ }^{(5)}$.

A associação do PNIF com sinais e sintomas de rinite foi investigada em 283 adultos, empregando-se para tanto a rinoscopia anterior, para avaliar os sinais e um questionário, que buscou investigar os sintomas. O PNIF mostrou-se fortemente associado aos sinais de rinite; entretanto, não foi verificada correlação com os sintomas, apesar dos valores se apresentarem muito próximos ao ponto de corte $(\mathrm{p}=0,057)$. Verificaram-se ainda diferenças nos valores do PNIF entre os participantes categorizados como assintomáticos, com rinite leve e com rinite moderada/grave, considerando-se tanto a classificação baseada nos sinais como também a realizada pelos sintomas. Os autores concluíram que o PNIF relaciona-se com os sinais de rinite, fornecendo, todavia, informação qualitativamente diferente da obtida por meio de questionários ${ }^{(2)}$. Outros estudos já revelaram ser comum observar inconsistências entre os sintomas de obstrução e o aspecto das cavidades nasais ${ }^{(8)}$.

Um estudo foi conduzido com 2523 pacientes que buscaram avaliação otorrinolaringológica por apresentarem queixas de problemas nasais crônicos ou do sono e teve por objetivo comparar a sensação de obstrução nasal, verificada por meio de escala visual analógica (NO-VAS), com parâmetros objetivos de avaliação. Os aspectos anatômicos referentes ao espaço nasal foram investigados empregando-se a rinometria acústica e os fisiológicos por meio do PNIF. A sensação de obstrução nasal apresentou associação com o PNIF e com quatro dos cinco parâmetros investigados na rinometria acústica, mesmo quando ajustado a uma série de fatores de confusão. O estudo demonstrou alta associação entre a sensação de obstrução nasal e as medidas de volume, área e fluxo aéreo nasal. Os autores acreditam que tanto a rinometria acústica quanto o PNIF são instrumentos objetivos válidos para avaliar a obstrução nasal subjetiva. Entretanto, apesar da associação significativa, o coeficiente de correlação mostrou-se baixo, indicando que essas avaliações objetivas não predizem completamente a percepção de obstrução do paciente ${ }^{(8)}$.

Em outro trabalho buscou-se comparar, em 290 adultos indicados para polissonografia, os métodos de avaliação da via aérea - anatômico, por meio da rinometria acústica; fisiológico, empregando-se o PNIF, e subjetivo, tanto por meio de um questionário (NOSE), quanto pela escala visual analógica (VAS). Observou-se ausência de correlação entre as avaliações objetivas e subjetivas, bem como entre a rinometria acústica e o PNIF e forte correlação entre as duas avaliações subjetivas (NOSE e VAS). Considerando-se a pobre correlação entre as categorias de mensuração nasal anatômica, fisiológica e subjetiva, e que os métodos são considerados válidos e reprodutíveis, os autores concluíram que cada método de avaliação traz informações de diferentes aspectos da via aérea nasal, devendo ser considerados complementares e não contraditórios ${ }^{(1)}$.

Como limitações da técnica pode-se ressaltar que o PNIF depende do esforço máximo inspiratório do paciente, sendo sua reprodutibilidade diretamente dependente da cooperação do participante ${ }^{(5,8)}$, do fornecimento de instruções corretas e claras por parte do investigador e da realização de procedimentos padronizados ${ }^{(8)}$.

As características do fluxo aéreo nasal durante a máxima inspiração não representam as condições fisiológicas da respiração ${ }^{(9)}$. Além de se tratar de uma medida instantânea ${ }^{(7)}$, a avaliação só abrange a inspiração( ${ }^{(3)}$, sendo consideradas as duas narinas simultaneamente, o que não reflete a alternância na resistência nasal durante a respiração ${ }^{(9)}$. Outra desvantagem a ser considerada é a influência das vias aéreas inferiores ${ }^{(2,20)}$, assim, baixos valores medidos pelo PNIF podem ser decorrentes de uma função pulmonar comprometida.

Alguns cuidados devem ser tomados, uma vez que a não adaptação correta da máscara ou um vedamento labial incompleto irão interferir nos resultados alcançados ${ }^{(9)}$. A propensão ao colapso da válvula nasal durante a inspiração forçada também deve ser cuidadosamente observada ${ }^{(6)}$, uma vez que gera medidas baixas em indivíduos sem obstrução nasal. Buscando minimizar este problema, um estudo ${ }^{(24)}$ avaliou o impacto do uso de dois diferentes dilatadores nasais concomitantemente à medida do pico inspiratório, tendo encontrado resultados contraditórios.

Até o presente momento o fonoaudiólogo tem se utilizado de um outro método para complementar sua avaliação clínica: o espelho de Glatzel. Trata-se de um instrumento empregado para medir indiretamente a função nasal que consiste em uma superfície polida e graduada que condensa o vapor de água expirado $^{(25)}$.

Um estudo realizado com atletas teve como objetivo estudar o emprego do espelho de Glatzel na avaliação da permeabilidade nasal, antes e após o exercício físico. Foi observado aumento na área de condensação nasal após os primeiros 10 minutos e no segundo momento apenas a área direita não mostrou significância estatística. Concluiu-se, assim, que a avaliação objetiva por meio do espelho de Glatzel mostrou o efeito descongestionante do exercício físico ${ }^{(26)}$. 
Uma pesquisa foi delineada com o objetivo de avaliar os efeitos da associação entre a remoção de hábitos de sucção e a terapia miofuncional orofacial na ampliação da aeração nasal. A avaliação do fluxo de ar expirado foi realizada prétratamento para remoção dos hábitos e 60 e 180 dias após o tratamento, por meio do Espelho Nasal Milimetrado de Altmann, uma adaptação do espelho de Glatzel. Diante da análise dos resultados foi concluído que a terapia miofuncional orofacial após a remoção de hábitos de sucção melhora a função respiratória nasal ${ }^{(27)}$.

A capacidade do espelho em identificar mudanças após uma intervenção também foi investigada em um estudo cujo objetivo era identificar a modificação da aeração nasal após a realização de manobras de massagem e limpeza nasal. Assim, vinte crianças de quatro a 11 anos de idade, com diagnostico de rinite alérgica, foram submetidas à avaliação do fluxo respiratório por meio do Espelho Nasal Milimetrado de Altmann. Para análise da área embaçada no espelho foi utilizado o software Scion Image for Windows (Beta 4.0.2.), sendo realizada a mensuração da área de acordo com a transformação da escala de 32 pixels por $1 \mathrm{~cm}^{2}$. A média das medidas encontradas foi de $16,6 \mathrm{~cm}^{2}$ antes e $20,3 \mathrm{~cm}^{2}$ depois da limpeza. Comparandose os resultados encontrados considerou-se significativo o aumento da aeração nasal após manobras de massagens de limpeza nasal ${ }^{(28)}$.

O espelho de Glatzel, apesar de muito utilizado na prática clínica fonoaudiológica, demonstrou, em pesquisa conduzida com crianças, ser um instrumento confiável como auxiliar diagnóstico apenas nos casos de grande obstrução nasal ${ }^{(29)}$.

\section{DISCUSSÃO}

De acordo com os dados levantados pode-se verificar que o Peak Nasal Inspiratory Flow tem sido empregado com frequência tanto em pesquisas quanto na prática médica ${ }^{(6,7,10-19)}$. O instrumento, por apresentar ${ }^{(2,5,5,19)}$ boa sensibilidade, especificidade e reprodutibilidade, baixo preço e demanda de tempo, poderia também ser considerado um método a ser empregado na Fonoaudiologia. Entretanto pesquisas precisam ser realizadas para se confirmar ou refutar essa hipótese.

Acredita-se que o instrumento poderia favorecer, na medida em que permite a obtenção de parâmetros mensuráveis, o controle clínico dos pacientes respiradores orais/oronasais ao longo da intervenção terapêutica. Assim, a evolução do quadro clínico, especialmente daqueles que apresentam rinite alérgica, poderia ser monitorada em cada sessão, uma vez que o PNIF tem como indicação a avaliação de resultados terapêuticos $^{(8)}$. De acordo com a literatura o PNIF pode ser usado no follow-up de alterações nasais ${ }^{(7)}$. O instrumento também poderia funcionar como um mecanismo de feedback na terapia miofuncional orofacial, como atualmente é realizado com o espelho de Glatzel $^{(28)}$, tornando-se uma ferramenta terapêutica. Um aspecto positivo neste caso é o fato de, considerando-se sujeitos antes e após alguma intervenção, os dados do PNIF serem altamente confiáveis ${ }^{(4)}$.

Outro emprego que poderia ser realizado pela Motrici- dade Orofacial seria nos casos de pesquisas. As informações sobre sintomas obstrutivos podem ser facilmente obtidas dos pacientes, entretanto, as evidências objetivas de um exame clínico realizado por um otorrinolaringologista, nem sempre estão disponíveis ${ }^{(2)}$. O PNIF, obviamente, não substitui a avaliação otorrinolaringológica, indispensável à condução de qualquer caso de respiração oral/oronasal. Mesmo porque em estudo com pacientes que apresentavam importante quadro obstrutivo, 25\% dos casos não foram detectados pelo $\mathrm{PNIF}^{(9)}$. Entretanto, há situações, como as pesquisas realizadas sem a participação de otorrinolaringologistas, em que o instrumento poderia se caracterizar como um dado objetivo respaldando, juntamente com a anamnese e a avaliação miofuncional orofacial, a impressão diagnóstica acerca do modo respiratório. A indicação do PNIF como um instrumento útil para screening populacional $^{(2)}$ respalda essa suposição. Métodos diferentes que avaliam dimensões distintas da obstrução nasal trazem informações de diferentes aspectos da via aérea, devendo ser considerados complementares ${ }^{(1)}$.

Ressalta-se também os casos de pacientes em atendimento fonoaudiológico pelo Sistema Único de Saúde com suspeita de respiração oral/oronasal que podem precisar aguardar longo tempo para conseguir a avaliação médica. Infelizmente na realidade do nosso país a integralidade no atendimento ao paciente é difícil de ser articulada na prática. Assim, o dado objetivo, nesse caso, também poderia auxiliar na decisão pela permanência ou não do paciente no Serviço enquanto aguarda o parecer do otorrinolaringologista, uma vez que geralmente o usuário já permaneceu por um longo tempo em uma fila de espera até ser chamado pela Fonoaudiologia. A boa sensibilida$\mathrm{de}^{(9)}$, especificidade ${ }^{(9)}$ e reprodutibilidade ${ }^{(2,5,19)}$ do instrumento auxiliariam nesse aspecto. Entretanto, cabe ressaltar que, ao menos nos casos de rinite alérgica, um baixo valor no PNIF pode não indicar uma alteração moderada/grave ${ }^{(2)}$.

Como qualquer exame complementar o PNIF, caso se comprove sua aplicabilidade na Motricidade Orofacial, deverá integrar a avaliação clínica conduzida pelo fonoaudiólogo, agregando dados, nunca os substituindo. Atualmente os profissionais já buscam realizar procedimentos que possam contribuir com dados objetivos sobre possíveis obstruções nasais, utilizando neste caso outro instrumento: o espelho de Glatzel ${ }^{(26-28)}$. Entretanto, o Peak Nasal Inspiratory Flow parece ser a ferramenta de uso simples mais empregada tanto na clínica quanto e em pesquisas, merecendo atenção também por parte do fonoaudiólogo.

\section{COMENTÁRIOS FINAIS}

De acordo com a revisão de literatura realizada verificou-se fortes indícios de que o Peak Nasal Inspiratory Flow, apesar de algumas limitações, é um instrumento útil na avaliação da patência nasal.

Estudos e pesquisas utilizando esse instrumento poderiam fornecer dados para a complementação do diagnóstico da obstrução nasal, auxiliando o fonoaudiólogo no atendimento a pacientes com distúrbios miofuncionais orofaciais e cervicais. 


\begin{abstract}
The speech-language pathologists that work in the Orofacial Myology field frequently have patients with nasal obstruction; however, the access to the professional responsible for the diagnosis can be difficult at times. The Peak Nasal Inspiratory Flow is a cheap and easy to handle instrument, broadly cited in the international literature, that has the aim to evaluate nasal patency. The aim of this study was to review the current literature regarding the Peak Nasal Inspiratory Flow, enabling considerations about its use in cervical and orofacial myology disorders. The literature review consulted Medline, Cochrane Library, LILACS and SciELO databases, using the keywords: peak, inspiratory, nasal and flow. The results showed that Peak Nasal Inspiratory Flow is a simple, cheap, validated technique with good reproducibility. Thus, it seems to be an useful instrument to be used in nasal patency evaluation, despite some limitations, providing complementary data to orofacial myofunctional diagnosis. However clinical trials are needed to prove this hypothesis.
\end{abstract}

Keywords: Diagnostic techniques, respiratory system; Permeability; Nasal obstruction/physiopathology; Mouth breathing; Nasal cavity/physiology

\title{
REFERÊNCIAS
}

1. Starling-Schwanz R, Peake HL, Salome CM, Toelle BG, Ng KW, Marks GB, et al. Repeatability of peak nasal inspiratory flow measurements and utility for assessing the severity of rhinitis. Allergy. 2005;60(6):795-800.

2. Nathan RA, Eccles R, Howarth PH, Steinsvåg SK, Togias A. Objective monitoring of nasal patency and nasal physiology in rhinitis. J Allergy Clin Immunol. 2005;115(3 Suppl 1):S442-59. Review.

3. Lam DJ, James KT, Weaver EM. Comparison of anatomic, physiological, and subjective measures of the nasal airway. Am J Rhinol. 2006;20(5):463-70.

4. Ottaviano G, Scadding GK, Coles S, Lund VJ. Peak nasal inspiratory flow; normal range in adult population. Rhinology. 2006;44(1):32-5.

5. Bermüller C, Kirsche H, Rettinger G, Riechelmann H. Diagnostic accuracy of peak nasal inspiratory flow and rhinomanometry in functional rhinosurgery. Laryngoscope. 2008;118(4):605-10.

6. Kjærgaard T, Cvancarova M, Steinsvåg SK. Does nasal obstruction mean that the nose is obstructed? Laryngoscope. 2008;118(8):1476-81.

7. Olivé Pérez A. La obstrucción nasal y su medida. Allergol Immunopathol. 2004;32(6):361-7.

8. Wheeler SM, Corey JP. Evaluation of upper airway obstruction - an ENT perspective. Pulm Pharmacol Ther. 2008;21(3):433-41.

9. Lee DK, Haggart K, Lipworth BJ. Reproducibility of response to nasal lysine-aspirin challenge in patients with aspirin-induced asthma. Ann Allergy Asthma Immunol. 2004;93(2):185-8.

10. Parikh AA, Scadding GK. Intranasal lysine-aspirin in aspirin-sensitive nasal polyposis: a controlled trial. Laryngoscope. 2005;115(8):1385-90.

11. Camargos P, Ibiapina C, Lasmar L, Cruz AA. Obtaining concomitant control of allergic rhinitis and asthma with a nasally inhaled corticosteroid. Allergy. 2007;62(3):310-6. Comment in: Allergy. 2007;62(3):213-5. Allergy. 2007;62(10):1214.

12. Høyvoll LR, Lunde K, Li HS, Dahle S, Wentzel-Larsen T, Steinsvåg SK. Effects of an external nasal dilator strip (ENDS) compared to xylometazolin nasal spray. Eur Arch Otorhinolaryngol. 2007;264(11):1289-94.

13. Galvan CCR, Caetano AJM. Efeito do treinamento dos músculos respiratórios sobre a função pulmonar no preparo pré-operatório de tabagistas. Acta Cir Bras. 2007;22(2):98-104.

14. Philpott CM, Conboy P, Al-Azzawi F, Murty G. Nasal physiological changes during pregnancy. Clin Otolaryngol Allied Sci. 2004;29(4):34351. Erratum in: Clin Otolaryngol Allied Sci. 2004;29(4):343-51. Erratum in: Clin Otolaryngol. 2005;30(1):88.

15. Philpott CM, El-Alami M, Murty GE. The effect of the steroid sex hormones on the nasal airway during the normal menstrual cycle. Clin Otolaryngol Allied Sci. 2004;29(2):138-42.
16. Wild DC, Philpott CM, Wolstenholme CR, Murty GE. Does hormone replacement therapy in post-menopausal women have any effect upon nasal physiology? J Laryngol Otol. 2008;122(7):707-10.

17. Held PA, Castro WM, Silva TLP, Silva KR, Di Lorenzo VAP. Treinamento muscular e da respiração nasal em crianças respiradoras orais. Fisioter Mov. 2008;21(4):119-27.

18. Serrano E, Klossek JM, Didier A, Dreyfus I, Sévenier F, Dessanges JF. Evaluation prospective de la méthode de mesure du flux inspiratoire nasal par le PNIF dans la rhinite allergique. Résultats de l'observatoire «Pratic en ORL». Rev Laryngol Otol Rhinol. 2007;128(3):173-7.

19. Dufour X, Gohler C, Delagranda A, Fontanel JP, Klossek JM. Peak Nasal Inspiratory Flow: apprentissage de la méthode de mesure et reproductibilité. Ann Otolaryngol Chir Cervicofac. 2007;124(3):115-9.

20. Ottaviano G, Lund VJ, Coles S, Staffieri A, Scadding GK. Does peak nasal inspiratory flow relate to peak expiratory flow? Rhinology. 2008;46(3):200-3.

21. Fonseca ACCF, Fonseca MTM, Rodrigues MESM, Lasmar LMLBF, Camargos PAM. Pico do fluxo expiratório no acompanhamento de crianças asmáticas. J Pediatr (Rio J). 2006;82(6):465-9.

22. Rossi DC, Munhoz DF, Nogueira CR, Oliveira TCM, Britto ATBO. Relação do pico de fluxo expiratório com o tempo de fonação em pacientes asmáticos. Rev CEFAC. 2006;8(4):509-17.

23. Papachristou A, Bourli E, Aivazi D, Futzila E, Papastavrou T, Konstandinidis T, et al. Normal peak nasal inspiratory flow rate values in Greek children and adolescents. Hippokratia. 2008;12(2):94-7.

24. Barnes ML, Lipworth BJ. Removing nasal valve obstruction in peak nasal inspiratory flow measurement. Ann Allergy Asthma Immunol. 2007;99(1):59-60.

25. Hungria H, Cruz AC. Otorrinolaringologia. 8a ed. Rio de Janeiro: Guanabara Koogan; 2000.

26. Calliari DS, Brescovici S, Kruse G. O espelho de Glatzel na avaliação da permeabilidade nasal antes e após exercício físico em indivíduos atletas [resumo]. Rev Soc Bras Fonoaudiol. 2005; Supl Especial.

27. Degan VV, Puppin-Rontani RM. Aumento da aeração nasal após remoção de hábitos de sucção e terapia miofuncional. Rev CEFAC. 2007;9(1):55-60.

28. Melo FMG, Cunha DA, Silva HJ. Avaliação da aeração nasal pré e pós a realização de manobras e massagens de limpeza nasal. Rev CEFAC. 2007;9(3):375-82.

29. Bassi IB, Franco LP, Motta AR. Eficácia do emprego do espelho de Glatzel na avaliação da permeabilidade nasal. Rev Soc Bras Fonoaudiol. 2009;14(3):367-71. 